\title{
Model of Infiltration of Spent Automotive Catalysts by Molten Metal in Process of Platinum Metals Recovery
}

\author{
Agnieszka Fornalczyk, Slawomir Golak, and Mariola Saternus \\ Faculty of Materials Engineering and Metallurgy, Silesian University of Technology, Krasinskiego 8, 40-019 Katowice, Poland \\ Correspondence should be addressed to Slawomir Golak; slawomir.golak@polsl.pl
}

Received 30 July 2013; Revised 30 September 2013; Accepted 30 September 2013

Academic Editor: Waqar Khan

Copyright ( 2013 Agnieszka Fornalczyk et al. This is an open access article distributed under the Creative Commons Attribution License, which permits unrestricted use, distribution, and reproduction in any medium, provided the original work is properly cited.

This paper presents the model for the washing-out process of precious metals from spent catalysts by the use of molten lead in which the metal flow is caused by the rotating electromagnetic field and the Lorentz force. The model includes the coupling of the electromagnetic field with the hydrodynamic field, the flow of metal through anisotropic and porous structure of the catalyst, and the movement of the phase boundary (air-metal) during infiltration of the catalyst carrier by the molten metal. The developed model enabled analysis of the impact of spacing between the catalysts and the supply current on the degree of catalyst infiltration by the molten metal. The results of calculations carried out on the basis of the model were verified experimentally.

\section{Introduction}

Spent catalysts containing precious metals are very attractive for recycling [1-6]. However, this process faces two major obstacles. First of all, a single catalyst carrier contains, at best, a few grams of platinum metals and secondly, the metals are trapped in the capillary structure of the ceramic carrier of a catalyst. That is why, the condition for economic viability of such a process is to minimise the cost of processing a single carrier. The authors have proposed and patented a new technology based on leaching PGMs from the catalyst structure by means of liquid metals [7]. This technique does not require a grinding of a catalyst carrier, thereby helping to reduce costs. Since the use of a mechanical pump in case of aggressive molten metal, in terms of large-serial recycling of catalysts, is practically impossible, the authors proposed a method based on a noncontact action of the electromagnetic field and magnetohydrodynamic phenomena $[8,9]$. Unfortunately, this method of propelling the metal is further complicated by the fact that the electromagnetic field acts on the metal outside of the catalyst carrier, which is the area where we would like to obtain the flow. The key for the success is to produce a pressure difference on both sides of the catalyst that is sufficient to overcome the resistance of the capillary structure of the catalyst carrier.
The technological process analyzed in the paper is quite a big challenge for modeling by means of computational fluid dynamics. So far only the flow of gases through a working automotive catalyst was modeled $[10,11]$. The modeling of the proposed recycling process of catalysts is much more complex. In the case of the considered process a multiphysical model taking into account coupling of the electromagnetic field with the hydrodynamic field has to be developed. Such models were previously developed for many industrial processes [12-15] but without a porous medium limiting the impact of electromagnetic field on the molten metal. Only paper [16] describes such a simple one-dimensional model of the Lorentz force infiltration of the porous structure.

At the initial state of the described process, there is a twophase flow of molten metal and air in the area of the catalyst carrier and during its infiltration the phase boundary moves. There are similar phenomena in the process of manufacturing metal matrix composites reinforced with a preform $[17,18]$. Created models of this process take into account the twophase flow and resistance of the preform to the penetration of the molten melt $[19,20]$. However, these models cannot be directly applied for the process of catalysts recycling because of the ultra-anisotropic, capillary structure of the catalyst carrier and nonuniform distribution of pressure on both sides of the carrier. In contrast to the process of the composite 
fabrication, the model of the analyzed recycling process must include not only the area of the porous carrier but also the whole device used in this process.

The multiphysical model presented in the paper was created on the basis of commercial software packages the Cedrat Flux (electromagnetic model) and the Ansys Fluent (hydrodynamic model). However, the coupling between the electromagnetic field and the hydrodynamic field was implement, with the use of software developed by the authors. The package Fluent has been extended with a new model allowing the analysis of two-phase flow through anisotropic, capillary structure taking into account the flow resistance and capillary pressure.

The paper contains description of the model of the catalyst carriers infiltration by molten metal, analysis of the influence of chosen parameters on efficiency of the process, and results of the experimental verification of the developed model.

\section{Model of Process}

The model of the analyzed process requires the coupling of the electromagnetic field with hydrodynamic field. It is possible to apply the commonly used one-way coupling of these fields [12-15] due to the fact that in the modeled process the magnetic Reynolds number is low; the moving (during the infiltration of catalyst carrier) phase boundary is present only in the area of the ceramic carrier and does not influence the distribution of the electromagnetic field because of zero macroscopic conductivity of the carrier capillary structure (independent of the presence of metal). This made it possible to divide simulation into two separate stages-the quasi-static simulation of the electromagnetic field giving the distribution of Lorentz force acting on liquid metal and the unsteady hydrodynamic simulation (Figure 1).

The research was conducted on the basis of twodimensional electromagnetic and hydrodynamic models. This allowed reduction of simulation time (to tens hours) with maintaining the representativeness of the model. It was possible because of the channel depth similar to the height of the catalyst carrier. It means that there was no flow under and above the carrier. At the same time the coil considerably higher than the channel allowed the assumption that the electromagnetic field is uniform in the direction parallel to the electromagnetic stirrer axis.

The periodicity of the electromagnetic field as well as the hydrodynamic field was assumed in the model (Figure 2). The size of the calculation domain expressed by $\beta$ angle depends on the number of catalyst carriers placed in the channel.

2.1. Channel Area outside Catalysts Carriers. The electromagnetic field was modeled using the equation, commonly used for quasistatic electromagnetic problems, based on the magnetic vector potential $[15,21,22]$. In the case of the two-dimensional model the potential is a scalar which significantly simplifies the calculations. Consider

$$
\nabla \times\left(\frac{1}{\mu} \nabla \times \mathbf{A}\right)+j \omega \sigma \mathbf{A}=\mathbf{J}_{s}
$$

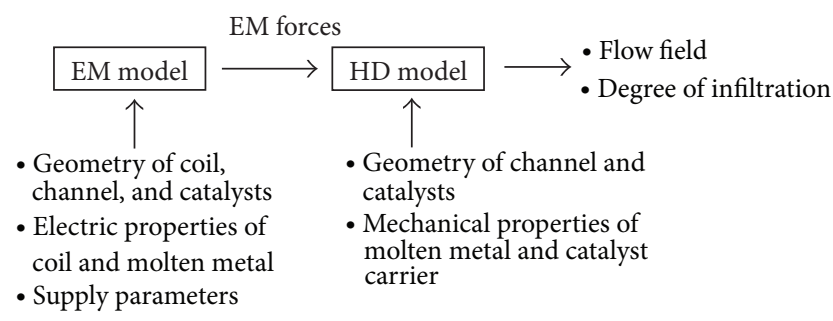

FIgURE 1: Schematic diagram of simulation.

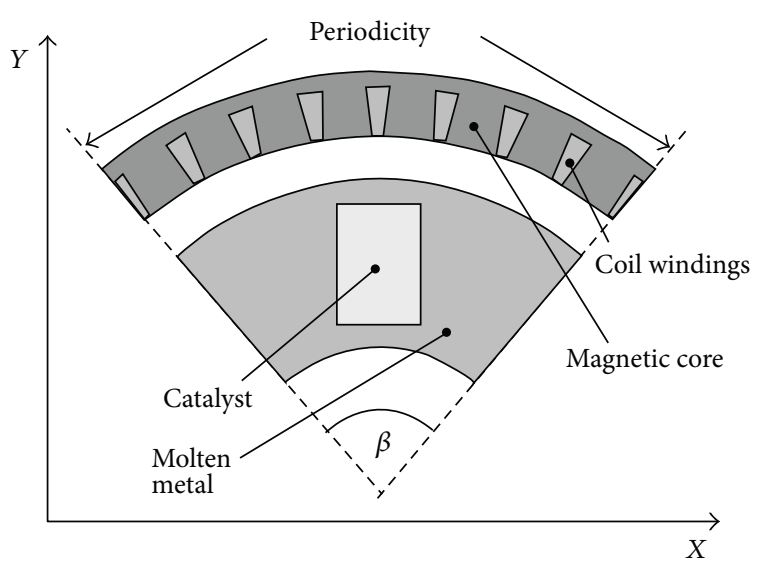

FIgURE 2: Schematic diagram of model geometry.

where $\mu, \sigma$ are magnetic permeability and conductivity of the molten metal, $\omega$ is angular frequency, $\mathbf{J}_{\mathbf{s}}$ is source current density.

After calculation of the potential distribution, it is possible to determine the Eddy current density $\mathbf{J}$ and the magnetic induction B. Consider

$$
\begin{aligned}
& \mathbf{B}=\nabla \times \mathbf{A}, \\
& \mathbf{J}=j \omega \sigma \mathbf{A} .
\end{aligned}
$$

On this basis, the distribution of the time-averaged Lorentz force acting on the molten metal was determined. Consider

$$
\mathbf{f}_{\mathbf{e}}=\frac{1}{2} \operatorname{Re}\left(\mathbf{J} \times \mathbf{B}^{*}\right) .
$$

The electromagnetic model was implemented by use of the commercial software Cedrat Flux complemented by the module which enables the calculation of the time-averaged Lorentz force.

The model of metal flow in the channel outside catalysts carriers was based on the solution of continuity and Navier-Stokes equations for the incompressible fluid. The electromagnetic force density $\mathbf{f}_{\mathbf{e}}$ was added as the source term in the momentum equation (5). Consider

$$
\nabla \cdot \mathbf{v}=0
$$

$$
\rho_{f}\left(\frac{\partial \mathbf{v}}{\partial t}+\mathbf{v} \cdot \nabla \mathbf{v}\right)=-\nabla p+\eta_{e} \nabla^{2} \mathbf{v}+\mathbf{f}_{\mathbf{e}}
$$


where $\mathbf{v}$ is velocity; $\eta_{e}$ is effective viscosity taking into account turbulent viscosity determined for the commonly used $k-\varepsilon$ model $[23,24], \rho$ is density; $p$ is pressure.

Due to the fact that the air squeezed out from the catalyst carrier is almost immediately removed from the molten metal as a result of the buoyancy force, only one phase in this areathe molten metal-was assumed.

The hydrodynamic model was created with the use of the Ansys Fluent complemented by User-Defined Functions enabling import of the electromagnetic force distribution from the Cedrat Flux.

2.2. Catalyst Carrier Area. The capillary structure of the carrier requires substantial modification of the model describing the channel zone outside the catalyst carriers. First of all, because the macroscopic electrical conductivity of the ceramic carrier area is zero (in the direction of the eddy current flow), the Lorentz force does not act on the metal in this area. Therefore, the source term representing the electromagnetic force can be neglected in (5). The capillary structure of the catalyst forces the unidirectional flow in the direction of the capillaries. The orientation of the catalyst according to the coordinate system (Figure 2) allowed reducing calculation to only the horizontal component of the velocity, assuming the vertical component $v_{y}$ is equal to zero.

After immersion of the catalyst into the molten metal, the infiltration of its capillary structure by the metal begins. This is why, in the area of the catalyst carrier, a two-phase flow was modeled using the modified volume of fluid (VOF) method which allowed tracking of the interface between air and molten metal during the infiltration of the carrier porous structure. The authors' modification of the VOF method enables modeling the two-phase flow through ultraanisotropic, capillary structure of the catalyst, taking into account the flow resistance and capillary pressure.

The catalyst capillary structure inhibits the flow of molten metal. The pressure drop across the length of the capillary can be determined on the basis of Hagen-Poiseuille equation [10]:

$$
\frac{\Delta p}{L}=\frac{28.5}{d^{2}} \eta v
$$

where $\Delta p$ is pressure drop, $L$ is unit length, $d$ is hydraulic diameter of the capillary, $\eta$ is viscosity of metal, and $v$ is velocity.

At the same time the presence of channel walls separating different fluid streams results in the lack of the term directly expressing the internal friction of fluid in the momentum equation (8). This friction was taken into account indirectly in the term derived from the Hagen-Poiseuille equation (6).

The metal, which is infiltrating the ceramic structure of the catalyst, also meets the additional resistance in the form of the capillary pressure due to the wetting angle for the system of ceramics and metal greater than 90 degrees. Consider

$$
p_{c}=\frac{4 \sigma \cos \theta}{d}
$$

where $p_{c}$ is capillary pressure, $\sigma$ is surface tension, and $d$ is capillary diameter.
TABLE 1: Geometry and material properties.

\begin{tabular}{lc}
\hline External radius of channel & $0.139 \mathrm{~m}$ \\
Internal radius of channel & $0.61 \mathrm{~m}$ \\
Internal radius of coil & $0.149 \mathrm{~m}$ \\
Frequency of coil & $50 \mathrm{~Hz}$ \\
Lead density & $10540 \mathrm{~kg} \cdot \mathrm{m}^{-3}$ \\
Lead viscosity & $0.002154 \mathrm{~Pa} \cdot \mathrm{s}$ \\
Width of carrier & $40 \mathrm{~mm}$ \\
Height of carrier & $60 \mathrm{~mm}$ \\
Hydraulic diameter of capillary & $0.00085 \mathrm{~m}$ \\
Wetting angle of carrier ceramics & $120^{\circ}$ \\
$\left(\mathrm{Al}_{2} \mathrm{O}_{3}\right.$ ) by molten lead & \\
Surface tension of lead & $0.439 \mathrm{~N} \cdot \mathrm{m}^{-1}$ \\
\hline
\end{tabular}

The capillary pressure and flow resistance resulting from the Hagen-Poiseuille relationship were included in the Navier-Stokes equation

$$
\rho_{m} \frac{d v_{x}}{d t}=-\frac{d p}{d x}-\frac{28.5}{d^{2}} \eta_{m} v_{x}-\frac{4 \sigma \cos \theta}{d} \frac{d \alpha}{d x},
$$

where $\alpha$ expresses the volume fraction of metal and $\rho_{m}$ and $\eta_{m}$ are the effective density and viscosity expressed by formulas:

$$
\begin{aligned}
& \rho_{m}=\alpha \rho_{\text {lead }}+(1-\alpha) \rho_{\text {air }} \\
& \eta_{m}=\alpha \eta_{\text {lead }}+(1-\alpha) \eta_{\text {air }}
\end{aligned}
$$

Tracing the metal phase distribution by the use of VOF method required adding to the model another continuity equation for the volume fraction of the metal:

$$
\frac{\partial \alpha}{\partial t}+\frac{\partial\left(\alpha v_{x}\right)}{\partial x}=0
$$

This multiphase model for the capillary medium was implemented as an additional module of the Fluent by the use of User-Defined Functions.

\section{Numerical Simulation}

Computer simulations (based on the developed model of the process) helped to evaluate the effectiveness of rinsing the catalyst structure by the liquid metal. The calculations also allowed the assessment of the impact of inductor current, the number of catalysts in the channel (distance between them) on the efficiency of the process. The study was conducted using liquid lead as a rinsing medium, for the process parameters shown in Table 1.

The research began with the two catalysts in the channel. The liquid metal was treated by a rotating electromagnetic field generated by a coil energized with a three-phase current of $40 \mathrm{~A}$. The distribution of the obtained electromagnetic force density is shown in Figure 3.

Electromagnetic forces reach the maximum value at the outer wall of the channel and vanish exponentially as the distance from the wall increases. This means that the effective 


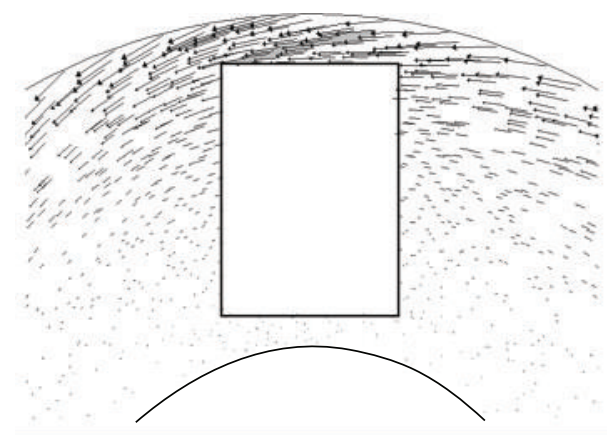

FIGURE 3: Distribution of electromagnetic force density.

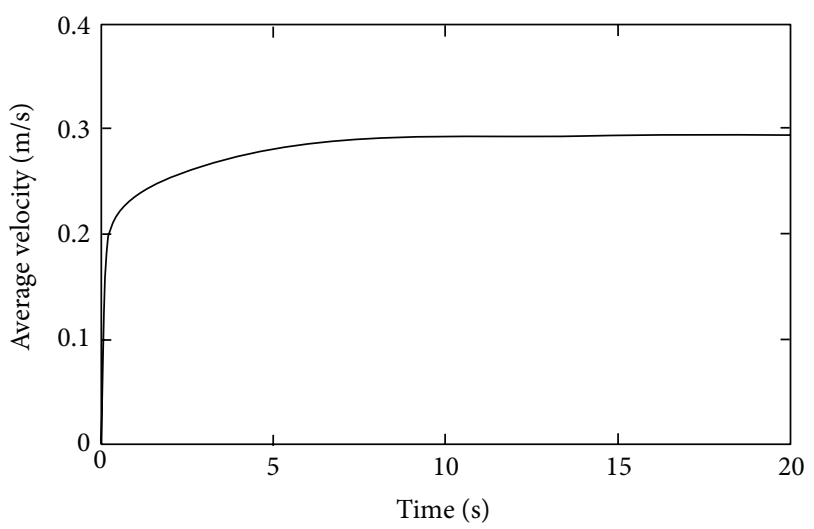

FIGURE 4: Average velocity as a function of time for two catalysts in the channel and current of $40 \mathrm{~A}$.

acceleration of metal occurs only in the outer metal layers. In the catalyst area, the Lorentz force does not act on the molten metal due to zero macroscopic conductivity of the carrier in the directions orthogonal to the direction of capillaries.

The distribution geometry of the electromagnetic force does not vary with changes in the supply current. The current only affects the values of this force.

About 20 seconds after power turn-on, the average velocity of the metal (Figure 4) reaches its maximum and thus the final flow structure and degree of the catalyst infiltration are obtained.

Only half of the carrier was infiltrated by metal, as shown in Figure 5. The main reason is insufficient, differential pressure between the inlet and outlet of capillaries which is not able to overcome the capillary pressure (7). As a result, precious metals are leached only from the half of the carrier. It dramatically worsens the efficiency and economy of the process.

Placing only one catalyst in the channel expands the area where the metal can be effectively accelerated, thereby increasing the pressure difference obtained on both sides of the catalyst. This results in a significant improvement of the catalyst structure infiltration (Figure 6). However, in the case of only one catalyst in the channel and approximately the same supply power, the efficiency of the process is reduced by half.

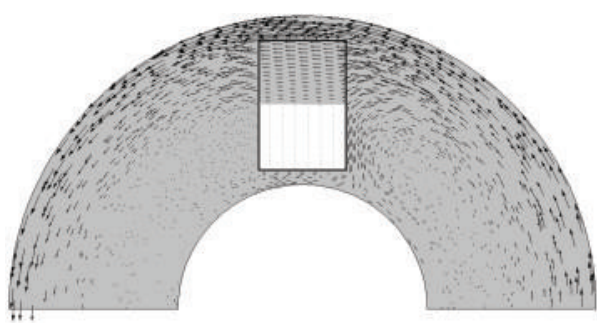

FIGURE 5: Distribution of velocity and degree of catalyst infiltration by liquid metal after stabilizing the flow field in the case of two catalysts in the channel and current of $40 \mathrm{~A}$.

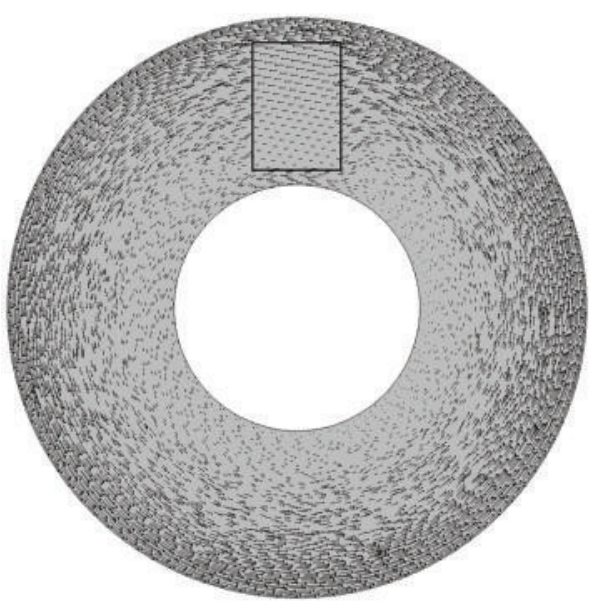

FIGURE 6: Distribution of velocity and degree of catalyst infiltration by liquid metal after stabilizing the velocity in the case of one catalyst in the channel and current of $40 \mathrm{~A}$.

For comparison, simulations for four and eight catalysts in the channel were carried out. However, in these cases there was almost no infiltration of the catalysts by liquid metal, which means the complete failure of the recycling process. All the energy was consumed for producing vortices in the spaces between the catalysts.

Increasing the supply current to $57 \mathrm{~A}$ and thus doubling the electromagnetic forces acting on the molten metal without changing the geometry of their distribution increased the average velocity that the liquid lead reaches in the channel (approximately $0.5 \mathrm{~m} / \mathrm{s}$ ). Increasing the difference of pressure between the inlet and outlet of the catalyst significantly improved the degree of infiltration of the carrier structure (Figure 8(a)).

However, the increase of the current causes a double increase in the power consumed by the device. That electrical efficiency of the process is halved. Comparison of the results obtained for one catalyst in the channel and the input current $40 \mathrm{~A}$ with the results for the two catalysts and supply current 57 A shows that better results can be obtained by increasing the spacing between the catalysts than by increasing the supply current (and consumed power). Increasing the supply current allowed a certain degree of infiltration of the carrier for case four (Figure 8(b)) and eight (Figure 8(c)) catalysts in the channel, but it should be considered as insufficient. 


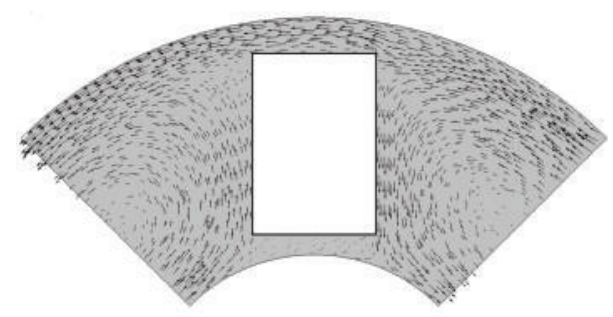

(a)

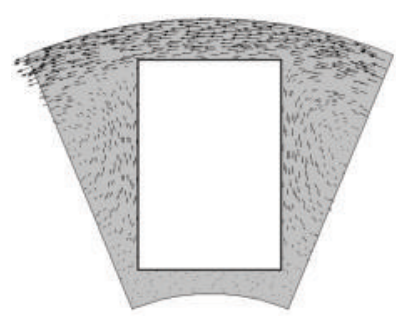

(b)

Figure 7: Distribution of velocity and degree of catalyst infiltration by liquid metal after stabilizing the velocity in the case of four (a) and eight (b) catalysts in the channel and current of $40 \mathrm{~A}$.

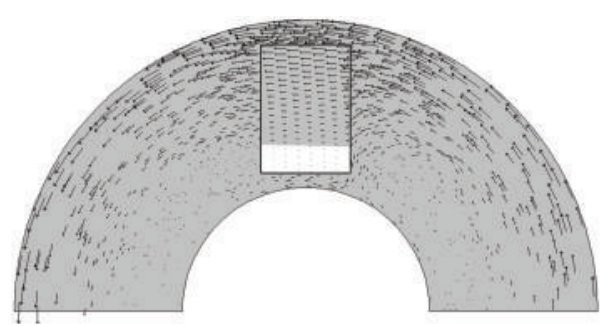

(a)

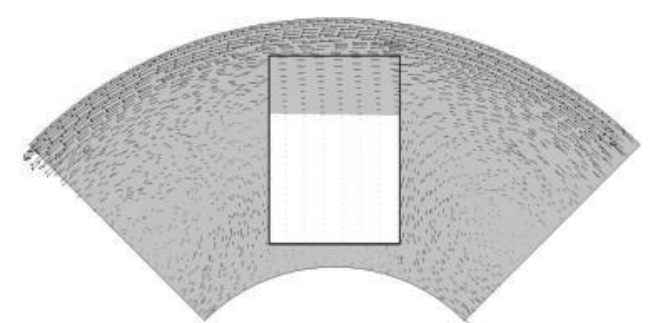

(b)

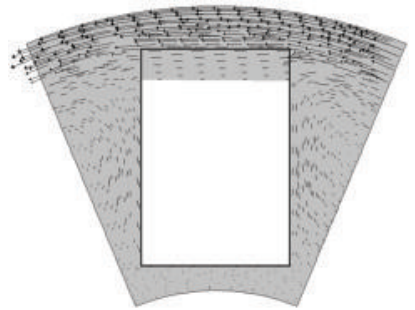

(c)

FIGURE 8: Distribution of velocity and degree of catalyst infiltration by liquid metal after stabilizing the velocity in the case of two (a), four (b), and eight (c) catalysts in channel and current of $57 \mathrm{~A}$.

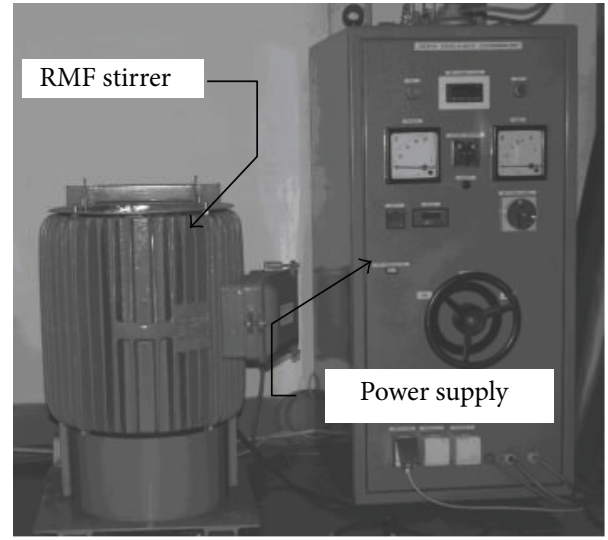

FIGURE 9: Experimental facility of automotive catalysts recycling.

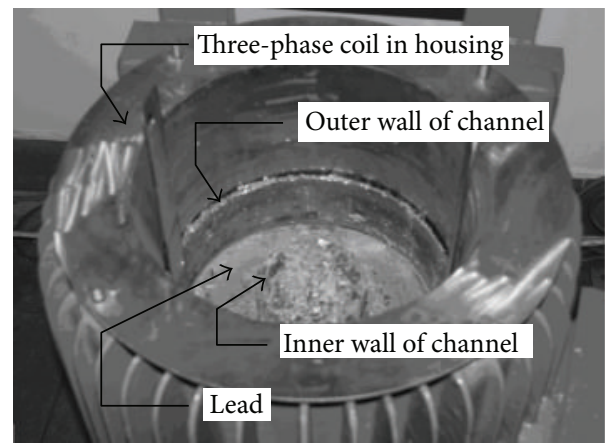

FIGURE 10: RMF stirrer.

\section{Experimental Verification}

The making of measurements of molten metal flow is not an easy task. A number of measurement methods were developed [25], but none of them is suitable for measuring the flow of metal through the porous structure of the catalyst. The only way to check the reliability of the presented model of infiltration is placing the catalysts in a stream of liquid metal, removing them after infiltration, and checking which capillaries were filled by the molten metal. The problem is that after the removal of the catalyst from the channel still liquid metal flows out from capillaries due to the action of the gravity and capillary pressure. For this reason, the only evidence of the presence of molten lead in a capillary during the process is the small amount of the solidified metal at its outlet.

Experimental verification of the described numerical model was carried with the use of the facility shown in Figure 9.

The facility consists of the rotating magnetic field stirrer (adapted a three-phase asynchronous motor) and the power supply (inverter). In the annular channel of the RMF stirrer (Figure 10) there was molten lead with a depth of 60 millimeters.

Three experiments were performed: for one, two, and four catalysts immersed in the molten lead. The three phase inductor of the stirrer was supplied with current of $40 \mathrm{~A}$ and frequency of $50 \mathrm{~Hz}$.

Front surfaces of the catalysts with remains of lead are presented in Figure 11 (the right wall of the catalyst is one close to the outer wall of the channel). The obtained experimental 


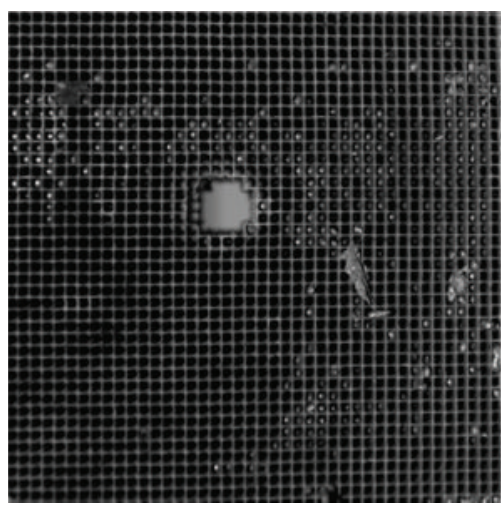

(a)



(b)

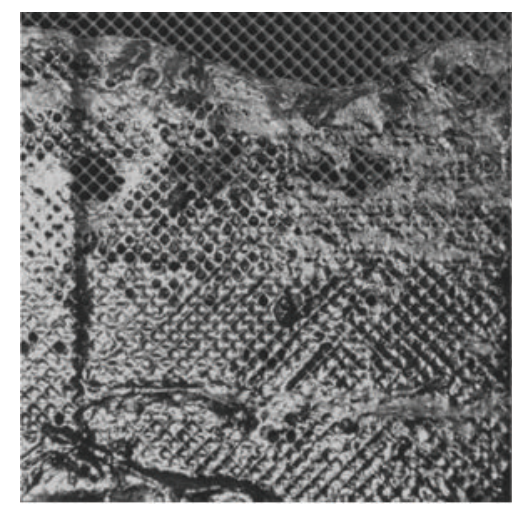

(c)

FIGURE 11: Front surface of catalyst carrier with remains of metal at outlets of capillaries: (a) four catalysts in channel, (b) two catalysts in channel, (c) one catalyst in channel.

results are consistent with the results of the numerical model presented in Figures 5-7. Vertical nonuniformity of metal distribution is the result of the three-dimensional character of the real process (that is not taken into account by the presented two-dimensional model), and imperfection of the used measurement method.

\section{Conclusions}

The experimentally verified numerical model of the catalyst infiltration by molten metal takes into account the most important phenomena present in the process-the magnetohydrodynamic flow, the two-phase flow, and the flow through porous medium. The developed model enables the analysis of the process, impossible to realize using only experimental methods.

The research indicated that in case of too high number of catalysts in the channel (too small distances between them) the molten metal did not have enough momentum to overcome the capillary pressure and the flow resistance in the catalyst carrier. If the current of coil increased, the situation would be better but at the cost of increased energy consumption on a single catalyst. Caulking spaces between a catalyst and channel walls could increase the pressure difference between the inlet and outlet of capillaries, but in case of a real technological process of recycling catalysts of random geometry, such a method is very difficult to implement.

The numerical simulations and experimental verification were conducted on a small laboratory scale. However, in case of industrial scale and higher diameters of the stirrer channel, the influence of the distance between catalysts and the supply current on the degree of carriers infiltration by the molten metal will be the same.

\section{References}

[1] M. Benson, C. R. Bennett, J. E. Harry, M. K. Patel, and M. Cross, "The recovery mechanism of platinum group metals from catalytic converters in spent automotive exhaust systems,"
Resources, Conservation and Recycling, vol. 31, no. 1, pp. 1-7, 2000.

[2] T. N. Angelidis, "Development of a laboratory scale hydrometallurgical procedure for the recovery of $\mathrm{Pt}$ and $\mathrm{Rh}$ from spent automotive catalysts," Topics in Catalysis, vol. 16-17, no. 1-4, pp. 419-423, 2001.

[3] B.-S. Kim, J.-C. Lee, S.-P. Seo, Y.-K. Park, and H. Y. Sohn, "A process for extracting precious metals from spent printed circuit boards and automobile catalysts," JOM, vol. 56, no. 12, pp. 55-58, 2004.

[4] Y. Kayanuma, T. H. Okabe, and M. Maeda, "Metal vapor treatment for enhancing the dissolution of platinum group metals from automotive catalyst scrap," Metallurgical and Materials Transactions B, vol. 35, no. 5, pp. 817-824, 2004.

[5] A. Fornalczyk and M. Saternus, "Removal of platinum group metals from the used auto catalytic converter," Metalurgija, vol. 48, no. 2, pp. 133-136, 2009.

[6] A. Fornalczyk and M. Saternus, "Vapour treatment method against other pyro- and hydrometallurgical processes applied to recover platinum from used auto catalytic converters," Acta Metallurgica Sinica, vol. 26, no. 3, pp. 247-256, 2013.

[7] A. Fornalczyk and M. Saternus, "Removal of platinum group metals from the used auto catalytic converter," Metalurgija, vol. 48, no. 2, pp. 133-136, 2009.

[8] A. Fornalczyk, R. Przylucki, M. Saternus, and S. Golak, "Analysis of using magneto-hydrodynamic pump for the platinum recovery from spent auto catalytic converters," Archives of Materials Science and Engineering, vol. 58, no. 2, pp. 199-204, 2012.

[9] A. Fornalczyk, S. Golak, and R. Przyłucki, "Analysis of electromagnetic and flow fields in the channel of a device used for precious metals leaching from auto catalytic converters," Przeglad Elektrotechniczny, vol. 89, no. 3, pp. 57-60, 2013.

[10] D. N. Tsinoglou, G. C. Koltsakis, D. K. Missirlis, and K. J. Yakinthos, "Transient modelling of flow distribution in automotive catalytic converters," Applied Mathematical Modelling, vol. 28, no. 9, pp. 775-794, 2004.

[11] Q. Su, L. Xie, S. Shuai, J. Wang, J. Song, and Z. Li, “Optimization of automotive catalytic converter by numerical modeling and simulation with detailed mechanism," Catalysis Today, vol. 216, pp. 292-298, 2013. 
[12] C. Sajdak, S. Golak, and A. Kurek, "Electromagnetic stirring of liquid ingot core in the process of continuous casting of steel," Przeglad Elektrotechniczny, vol. 83, no. 3, pp. 67-71, 2007.

[13] J. Barglik, D. Dołega, and A. Smagór, "Coupled temperature electromagnetic flow fields in the electromagnetic stirrer with a rotating magnetic field," Magnetohydrodynamics, no. 4, pp. 387392, 2010.

[14] M. Ščepanskis, A. Jakovičs, and E. Baake, "Statistical analysis of the influence of forces on particles in EM driven recirculated turbulent flows," Journal of Physics: Conference Series, vol. 333, no. 1, Article ID 012015, 2011.

[15] S. Golak and R. Przylucki, "Homogenization of electromagnetic force field during casting of functionally graded composites," IEEE Transactions on Magnetics, vol. 47, no. 12, pp. 4701-4706, 2011.

[16] R. M. Andrews and A. Mortensen, "Lorentz force infiltration of fibrous preforms," Metallurgical Transactions A, vol. 22, no. 12, pp. 2903-2915, 1991.

[17] M. Dyzia, A. J. Dolata, and J. Sleziona, "Preliminary analysis of aluminum matrix compositions for composites reinforcement with carbon fibers," Steel Research International, vol. 83, no. 10, pp. 981-987, 2012.

[18] A. Boczkowska, P. Chabera, A. J. Dolata, M. Dyzia, and A. Ozieblo, "Porous ceramic-metal composites obtained by infiltration methods," Metalurgija, vol. 52, no. 3, pp. 345-348, 2013.

[19] J. Xie, R. S. Amano, P. M. Mohan Das, and P. K. Rohatgi, “Transit simulation analysis for the pressure infiltration of aluminum melts into metal matrix components," in ASME Summer Heat Transfer Conference, pp. 213-220, July 2005.

[20] B. Wang and K. M. Pillai, "Numerical simulation of pressure infiltration process for making metal matrix composites using dual-scale fabrics," Metallurgical and Materials Transactions A, 2013.

[21] P. Karban, V. Kotlan, and I. Dolezel, "Numerical model of induction shrink fits in monolithic formulation," IEEE Transactions on Magnetics, vol. 48, no. 2, pp. 315-318, 2012.

[22] S. Golak and R. Zagorski, "Model and optimization of electromagnetic filtration of metals," Metalurgija, vol. 52, no. 2, pp. 215218, 2013.

[23] B. E. Launder and D. B. Spalding, "The numerical computation of turbulent flows," Computer Methods in Applied Mechanics and Engineering, vol. 3, no. 2, pp. 269-289, 1974.

[24] T. Merder, "Effect of casting flow rate on steel flow phenomena in tundish," Metalurgija, vol. 52, no. 2, pp. 161-164, 2013.

[25] S. Eckert, A. Cramer, and G. Gerbeth, "Velocity measurement techniques for liquid metal flows," in Magnetohydrodynamics, vol. 80 of Fluid Mechanics and Its Applications, pp. 275-294, Springer, Dordrecht, The Netherlands, 2007. 


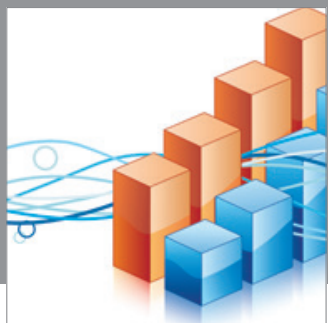

Advances in

Operations Research

mansans

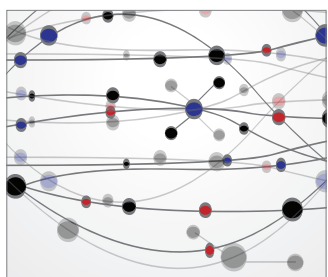

The Scientific World Journal
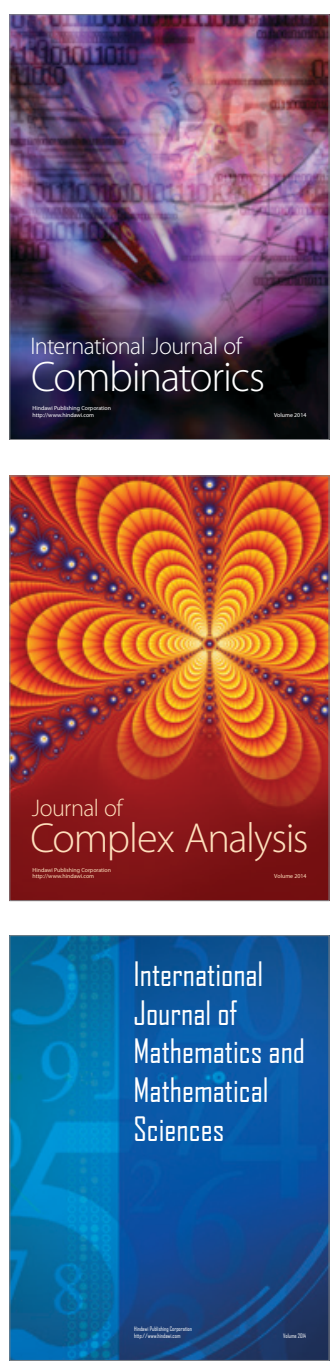
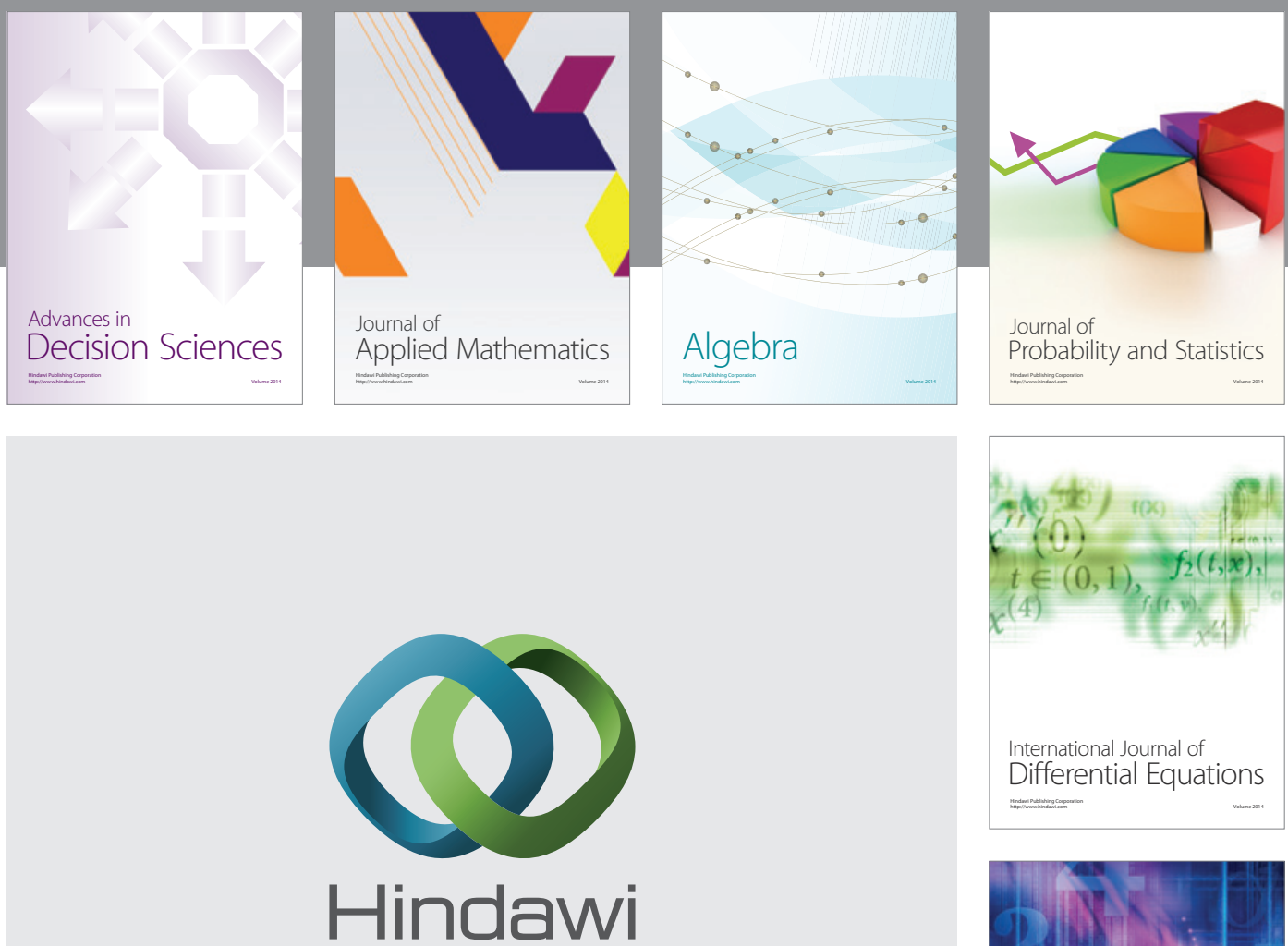

Submit your manuscripts at http://www.hindawi.com
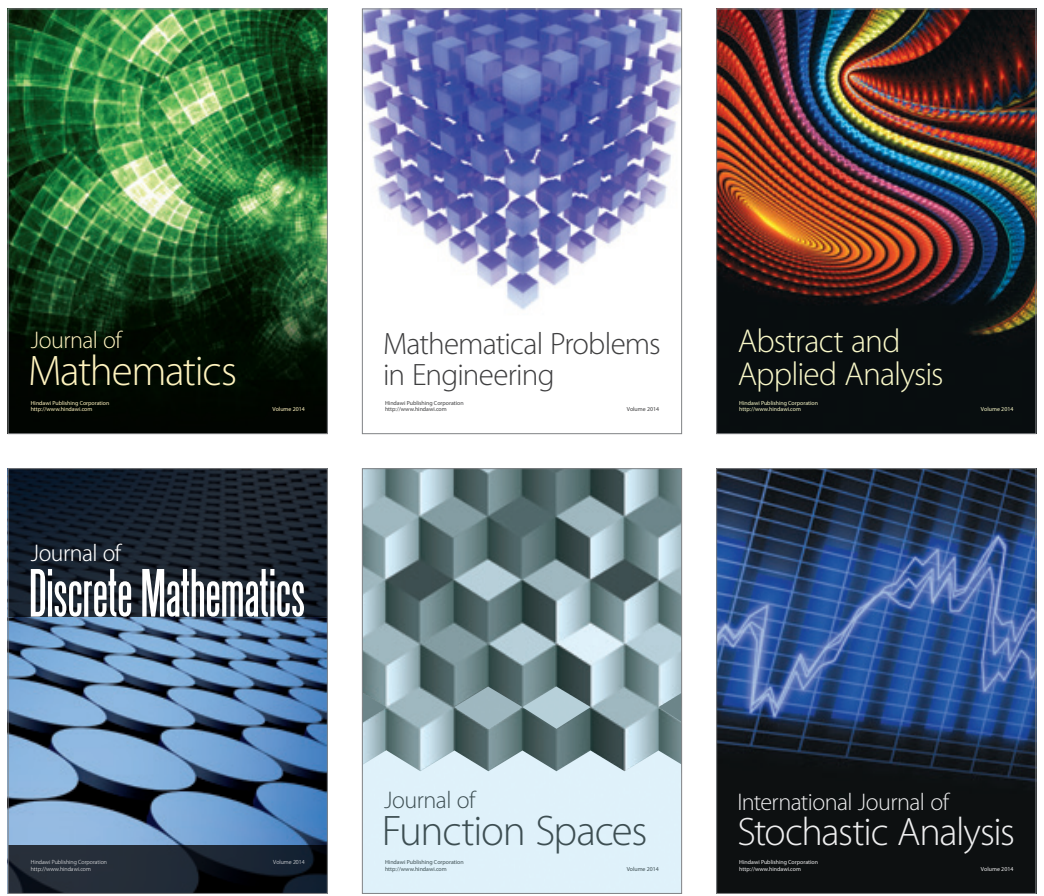

Journal of

Function Spaces

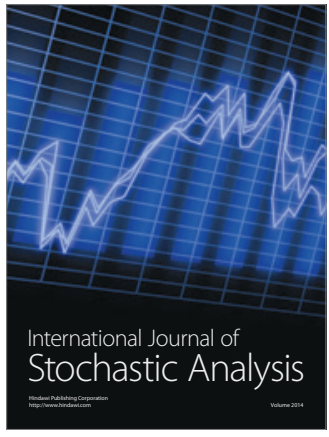

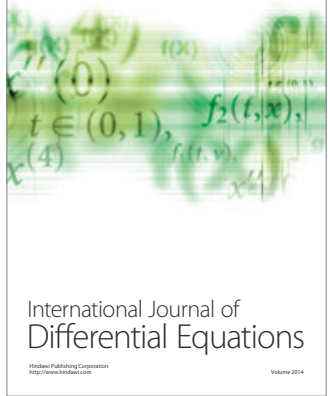
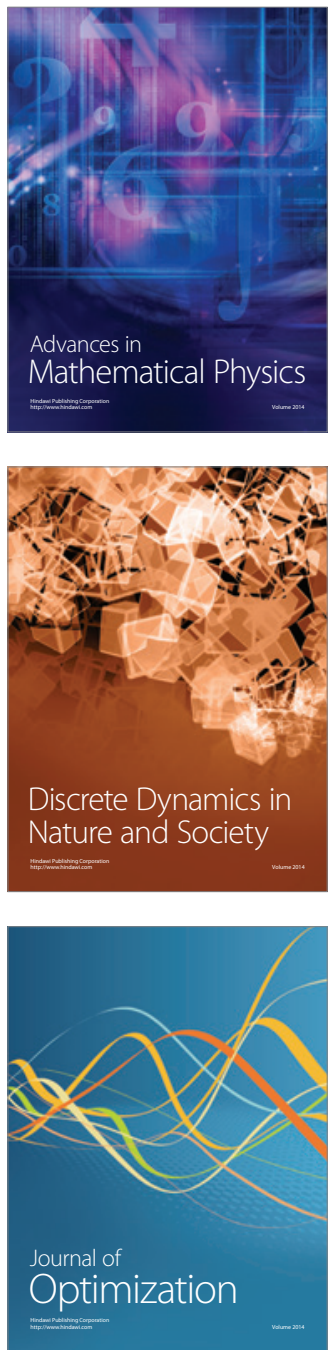